\title{
Impacto das emoções no desempenho acadêmico e na qualidade de vida dos
}

\section{estudantes de Medicina}

Impact of emotions on academic performance and quality of life of Medical students

Impacto de las emociones en el rendimiento académico y la calidad de vida de los estudiantes de

\section{Medicina}

\author{
Miguel Carlos Azevedo Cruz \\ ORCID: https://orcid.org/0000-0001-7719-9749 \\ Universidade Evangélica de Goiás, Brasil \\ E-mail: miguelcarlosac@hotmail.com \\ Eduardo Francisco Cardoso \\ ORCID: https://orcid.org/0000-0002-1609-1918 \\ Universidade Evangélica de Goiás, Brasil \\ E-mail: edu192375173@gmail.com \\ Thaís Ribeiro Garcia \\ ORCID: https://orcid.org/0000-0002-5658-4151 \\ Universidade Evangélica de Goiás, Brasil \\ E-mail: thaisrgarcia13@hotmail.com \\ Rafaela Melo Macedo \\ ORCID: https://orcid.org/0000-0001-8005-5236 \\ Universidade Evangélica de Goiás, Brasil \\ E-mail: melorafamed@gmail.com \\ Jalsi Tacon Arruda \\ ORCID: https://orcid.org/0000-0001-7091-4850 \\ Universidade Evangélica de Goiás, Brasil \\ E-mail: jalsitacon@gmail.com
}

\section{Resumo}

A Qualidade de Vida é um desafio para as práticas da saúde, ainda mais em tempos de pandemia da COVID-19. Ingressar no ensino superior, faz parte de uma das fases de transição na vida humana, e envolve uma série de adaptações. Os estudantes da área de saúde, em especial os de medicina por ser um curso integral que demanda muitas horas de dedicação aos estudos, sofrem muitas pressões que podem afetar o desempenho acadêmico. Assim, o presente estudo reúne informações sobre os fatores emocionais que influenciam a qualidade de vida dos acadêmicos de medicina, no formato de uma revisão integrativa. Buscas foram realizadas nas bases de dados PubMed, SciELO, BVS e Google Acadêmico, utilizando os descritores: "Qualidade de Vida" AND "Estudantes de Medicina" por estudos sobre esse tema. As análises confirmam que as pressões da vida acadêmica podem prejudicar o desenvolvimento curricular dos estudantes, seja por afetar a cognição ou as habilidades práticas, além de reduzir o senso de confiança e inibir as relações interpessoais e expressão emocional. Dificuldade de atenção durante as aulas, não pensar com clareza, dificuldade de foco, incapacidade de completar tarefas, risco de reprovação e potencial desistência acadêmica, aumentam consideravelmente nesses casos. Tais fatores, quando presentes, são importantes indicadores de sofrimento emocional e impactam consideravelmente na qualidade de vida do acadêmico de medicina.

Palavras-chave: COVID-19; Educação Médica; Emoções; Indicadores de Qualidade de Vida.

\begin{abstract}
Quality of Life is a challenge for health practices, even more so in times of the COVID-19 pandemic. Entering higher education is part of one of the transition phases in human life and involves a series of adaptations. Students in the health field, especially those in medicine, as it is an integral course that requires many hours of dedication to their studies, suffer many pressures that can affect academic performance. Therefore, this study gathers information about the emotional factors that influence the quality of life of medical students in the form of an integrative review. We performed in PubMed, SciELO, BVS, and Academic Google databases using the descriptors: "Quality of Life" AND "Medical Students" for studies on this topic. The analyzes confirm that the pressures of academic life can hinder students' curricular development, either by affecting cognition or practical skills, in addition to reducing the sense of trust and inhibiting interpersonal relationships and emotional expression. Difficulty in paying attention during classes, not thinking, difficulty focusing, inability to complete tasks, risk of failure, and potential academic dropout increase considerably in these cases. Such factors, when present, are important indicators of emotional distress and have a considerable impact on the quality of life of medical students.
\end{abstract}

Keywords: COVID-19; Medical Education; Emotions; Quality of Life Indicators. 


\begin{abstract}
Resumen
La calidad de vida es un desafío para las prácticas de salud, especialmente en tiempos de la pandemia de COVID-19. El ingreso a la educación superior es parte de una de las fases de transición en la vida humana e implica una serie de adaptaciones. Los estudiantes del campo de la salud, especialmente los de medicina, por ser un curso integral que requiere muchas horas de dedicación a sus estudios, sufren muchas presiones que pueden afectar el rendimiento académico. Así, este estudio recoge información sobre los factores emocionales que influyen en la calidad de vida de los estudiantes de medicina, en forma de revisión integradora. Las búsquedas se realizaron en las bases de datos PubMed, SciELO, BVS y Academic Google utilizando los descriptores: Calidad de vida AND Estudiantes de medicina; por estudios sobre este tema. Los análisis confirman que las presiones de la vida académica pueden obstaculizar el desarrollo curricular de los estudiantes, ya sea al afectar la cognición o las habilidades prácticas, además de reducir el sentido de confianza e inhibir las relaciones interpersonales y la expresión emocional. La dificultad para prestar atención durante las clases, no pensar con claridad, la dificultad para concentrarse, la incapacidad para completar las tareas, el riesgo de reprobar y la potencial deserción académica aumentan considerablemente en estos casos. Dichos factores, cuando están presentes, son indicadores importantes de angustia emocional y tienen un impacto considerable en la calidad de vida de los estudiantes de medicina.
\end{abstract}

Palabras clave: COVID-19; Educación Médica; Emociones; Indicadores de Calidad de Vida.

\title{
1. Introdução
}

A Organização Mundial da Saúde define Qualidade de Vida (QV), como sendo a percepção do indivíduo sobre sua vida, seu contexto cultural e social, seus padrões e valores, levando em consideração seus objetivos, expectativas e preocupações englobando as perspectivas e o grau de satisfação encontrado na vida familiar, afetiva, social e ambiental (WHO, 2012). Envolve o bem-estar espiritual, físico, mental, psicológico e emocional, além de relacionamentos sociais, como família e amigos, saúde, educação, habitação, saneamento básico e outras circunstâncias da vida (Cruz et al., 2021). A QV é amplamente discutida em diversos cenários. Nos mais recentes, a QV é um desafio para as práticas da área da saúde, ainda mais em tempos de pandemia da COVID-19. Dentro desses cenários, o ensino superior tornou-se um campo de estudo, visto que os universitários são submetidos a diferentes níveis de pressões durante o período acadêmico, sendo imprescindível analisar os aspectos que podem influenciar a qualidade de vida dessas pessoas (Chazan et al., 2015).

Um conceito multifatorial, sobre o bem-estar global do indivíduo, pode ser avaliado nos quesitos da QV envolvendo dimensões fundamentais de ordem física, social, emocional e, dimensões específicas, tais como cognição, sexualidade, produtividade pessoal, desconforto e dor, considerando a percepção do indivíduo e suas relações com o meio ambiente (Leite et al., 2020). As investigações sobre QV fomentam discussões sob distintas perspectivas teóricas e recortes metodológicos (Silva et al., 2020; Araújo et al., 2021).

O cotidiano acadêmico pode ser analisado na perspectiva da QV dos universitários (Anversa et al., 2018). Ingressar no ensino superior faz parte da transição da adolescência para a fase de adulto jovem, o que traz repercussões para o desenvolvimento psicológico dos estudantes, representando a primeira tentativa de uma identidade autônoma e própria do ser humano (Dutra et al., 2021). No primeiro ano na universidade, o estudante precisa lidar com uma série de transformações que envolvem, desde o seu futuro profissional até o estabelecimento de novas redes de interação social, inclusive conhecer pessoas e conviver durante as aulas, interagir entre si durante a realização de trabalhos acadêmicos. Por outro lado, quando não há essa nova formação de vínculos de amizades, os acadêmicos sentem-se inibidos e contam apenas com os próprios recursos psicológicos e o apoio das antigas amizades anteriores ao ingresso a universidade, as quais podem estar distantes nesse momento (Mendes \& Dias, 2021).

Outro detalhe importante a ressaltar é que muitos desses universitários, ao buscar o ensino superior, deixam sua cidade natal para residir longe dos familiares, fator que pode influenciar na adaptação à realidade da formação profissional (Ferreira et al., 2020). Dessa forma, permanecer e finalizar o ensino superior merece um destaque especial, uma vez que exige um intenso processo de envolvimento do estudante na sua formação (Anversa et al., 2018). A vida acadêmica, de forma geral, espera que 
esse universitário tenha autonomia para a sua aprendizagem, na administração do seu tempo e na definição de metas e estratégias para os estudos (Bezerra et al., 2020). Mas, muitas das vezes, os estudantes simplesmente não sabem como agir diante das dificuldades do mundo acadêmico (Mazucato et al., 2020). E além das aulas, estágios, seminários trabalhos de conclusão de curso, há uma preocupação constante com a vida pós-formatura, gerando ansiedade e insegurança.

Dessa maneira, novas pesquisas precisam explorar quais os fatores que influenciam a QV dos estudantes da área de saúde, em especial os de medicina por ser um curso integral que demanda muitas horas de dedicação aos estudos. Sendo assim, o presente estudo reúne informações sobre os fatores emocionais que influenciam a qualidade de vida dos acadêmicos de medicina, no formato de uma revisão integrativa, que tem por finalidade sintetizar resultados obtidos em diferentes pesquisas, de maneira ordenada e abrangente, integrando o conhecimento sobre o tema.

\section{Metodologia}

O presente estudo é uma revisão integrativa da literatura, compreendendo as seguintes etapas: identificação do tema e formulação da questão norteadora da pesquisa, elaboração dos critérios de inclusão e exclusão na seleção dos artigos, construção dos instrumento para buscas e coleta de dados relevantes dos estudos encontrados, categorização e avaliação dos estudos selecionados, análise e interpretação dos dados obtidos nos artigos, e apresentação da revisão/síntese dos conhecimentos.

A questão norteadora da presente pesquisa foi: "Quais são os fatores emocionais que influenciam na qualidade de vida dos acadêmicos de medicina?" Para responder tal questionamento, foram realizadas buscas para obter estudos sobre o tema no banco de dados do PubMed, na Biblioteca Eletrônica Científica On-line (SciELO - Scientific Eletronic Library Online), Biblioteca Virtual em Saúde (BVS) e no mecanismo virtual de pesquisa do Google Acadêmico. Foram utilizados os seguintes descritores do Ciências da Saúde (DeCS), que constam na plataforma DeCS/MeSH: "Qualidade de Vida" e "Estudantes de Medicina", utilizados em português e inglês, em combinação com auxílio do operador Booleano (AND). A coleta de dados foi realizada entre maio e julho de 2021. A escolha dessas bases ocorreu em razão da amplitude, no que tange à abrangência dos periódicos indexados que atuam na temática discutida.

Os critérios de inclusão foram: artigos originais ou de revisão e relatos de casos disponíveis completos que investigaram a relação dos fatores emocionais e sua influência na vida dos acadêmicos de medicina; sem restrição de data de publicação e publicados nos idiomas português e inglês. Foram excluídos estudos que não investigassem o tema proposto, artigos de opinião, carta ao editor, estudos realizados com estudantes que não fossem da área da saúde/medicina, ou estudantes do ensino fundamental e médio; e que não atendessem aos critérios de inclusão descritos.

A primeira etapa de seleção foi identificar e retirar as duplicatas, após isso analisar os potenciais estudos e se preenchiam os critérios estabelecidos, passando por uma seleção inicial das publicações realizada com leitura do título e resumo para verificar se enquadravam na temática. Após essa etapa, os estudos previamente selecionados foram lidos na íntegra, empregando os critérios de inclusão e exclusão, para análise e obtenção das informações necessárias para a construção do presente estudo. Caso houvesse discordância entre os avaliadores sobre os critérios analisados, foi realizada discussão específica sobre o artigo em questão, confrontando ideias com base nos critérios adotados para a revisão. Ao final da seleção foram incluídos 43 estudos mais recentes publicados (Tabela 1). Os estudos incluídos na revisão integrativa permitem uma avaliação crítica do tema discutido sendo possível identificar lacunas que poderão direcionar futuras pesquisas. 
Tabela 1. Estudos encontrados nas buscas realizadas nas bases de dados.

\begin{tabular}{c|c|c}
\hline FONTES DE INFORMAÇÃO & ESTRATÉGIA DE BUSCA & RESULTADOS \\
\hline PubMed & $\begin{array}{c}\text { ("quality of life"[MeSH Terms] OR } \\
\text { ("quality"[All Fields] AND "life"[All } \\
\text { Fields]) OR "quality of life"[All Fields]) } \\
\text { AND ("students, medical"[MeSH Terms] } \\
\text { OR ("students"[All Fields] AND } \\
\text { "medical"[All Fields]) OR "medical } \\
\text { students"[All Fields] OR ("medical"[All } \\
\text { Fields] AND "students"[All Fields]) }\end{array}$ \\
BVS & $\begin{array}{c}2214 \\
\text { Google Acadêmico }\end{array}$ & 2041 \\
\hline
\end{tabular}

Fonte: Autores (2021).

\section{Resultados e Discussão}

Durante as análises dos estudos selecionados, percebe-se que as pressões da vida acadêmica podem prejudicar o desenvolvimento curricular dos estudantes, seja por afetar a cognição ou as habilidades práticas, além de reduzir o senso de confiança e inibir as relações interpessoais e expressão emocional (Christ et al., 2019). Os estudantes que sofrem de tais pressões apresentam motivação reduzida para comparecer as atividades, dificuldade de atenção durante as aulas, não pensam com clareza, possuem dificuldade de foco, são incapazes de completar suas tarefas. Nesse contexto, o risco de reprovação e potencial desistência acadêmica aumentam consideravelmente. Tais fatores, quando presentes, são importantes sugestivos de sofrimento emocional (Meinck et al., 2017).

Estudantes que estão submetidos à exaustão emocional, demonstram problemas de comportamento e tornam-se mais rebeldes e resistentes a professores e/ou seus colegas de classe. Exemplos de tais atitudes são brigas, semblante frio ou sem sentimentos (Mceachern et al., 2008; Wang et al., 2019). Além disso, não raro, verifica-se sentimentos de ansiedade ou fobia, visto que o acadêmico tem medo ou receio de situações que lhe causem desgaste. Não menos importante, a vivência durante a infância de situações de abuso emocional e abuso infantil têm grande efeito sobre a vida universitária podendo causar transtornos psicológicos como depressão e ansiedade (Rich et al., 1997; Madu et al., 2003).

Cazolari et al. (2020) realizaram um estudo na Universidade Federal de São Paulo - UNIFESP, com mais de 300 acadêmicos de medicina, e perceberam que $65 \%$ deles apresentaram exaustão emocional, cuja gravidade aumenta à medida que o curso progride. De forma a corroborar esses resultados, Santos et al. (2017), em estudo realizado na Universidade Estadual do Sudoeste da Bahia, verificaram que cerca de $32 \%$ dos 115 estudantes participantes pesquisados apresentaram suspeita de Transtorno Mental Comum, caracterizado por sintomas depressivos, estados de ansiedade, irritabilidade, fadiga, insônia, dificuldade de memória e concentração e queixas somáticas, além de manifestar-se como uma mistura de sintomas somáticos, ansiosos e depressivos. Como exposto por Silva et al. (2020), diversos são os fatores interferentes da QV de acadêmicos, sendo na esfera social o perfil psicológico e transtornos, na esfera saúde física o sedentarismo, na esfera econômica as bolsas e dívidas relacionadas à vida acadêmica.

Andrade et al. (2019), diferentemente, encontraram escores de melhora na qualidade de vida, à medida que o curso avança, obtendo médias de 45/100 durante o primeiro ano de curso e 62/100 para o quarto ano letivo. De acordo com exposto anteriormente, durante o último biênio do curso médico, o período de Internato (correspondendo do $9^{\circ}$ ao $12^{\circ}$ período acadêmico do curso), Meyer et al. (2010) observaram valores de QV acima de 60 em um questionário que abrange questões dentro dos 
domínios físico, psicológico, social e ambiental, sendo que esse escore varia de 0 a 100, e quanto mais próximo de 100 melhor a qualidade de vida.

As populações que mais frequentemente reportam abuso emocional são do sexo feminino, etnia branca e idade avançada (Brodski \& Hutz, 2012; Spadine et al., 2020). Em adição a isso, Vidourek (2017) relataram que, num período de um ano, mais de 1 em cada 10 acadêmicos sofreram pressões emocionais nos Campi. Portanto, como forma de proteção, muitos alunos excluem-se de relações interpessoais e por vezes mostram-se sem senso de humor e com afeto negativo. Dessa forma, suas habilidades para perceber, entender e expressar emoções estão bastante reduzidas ou ausentes.

Ao se aprofundar mais nas adversidades emocionais enfrentadas pelos acadêmicos de medicina, verifica-se que a síndrome de Burnout, conjunto de sinais e sintomas, cujo desfecho é a depleção física e mental do indivíduo, está bastante presente nessa população (Mitra et al., 2018). Tal síndrome sofre influência de fatores como o sexo, uma vez que mulheres apresentam maiores níveis de sintomas depressivos e de esgotamento que homens, e tempo de estudo, sendo o Burnout menos prevalente quanto maior o nível de experiência dos acadêmicos (Grace, 2018; El-Ibiary et al., 2017). A prevalência dessa síndrome em graduandos da área da saúde (enfermagem, farmácia e medicina), varia de 4 a 7 em cada 10 acadêmicos, intervalo que se explica pelo uso de diferentes tipos de escores e métodos de pesquisa. Nesse contexto, é importante salientar que o momento de pandemia da COVID-19, parece relacionar-se com uma maior prevalência da síndrome de Burnout, porém mais estudos são necessários para aumentar o nível de evidência dessa informação e excluir possíveis vieses (Shrestha et al., 2021).

Um estudo coreano que analisou o modelo virtual de estudo, percebeu que o fluxo de aprendizagem, definido como processo psicológico de aprendizagem, sofre influências da síndrome de Burnout. Estudantes coreanos com maior fluxo de aprendizagem apresentaram menores índices de Burnout (Im \& Lee, 2021). Além disso, o empoderamento intelectual e a inteligência emocional também parecem ser fatores que reduzem o esgotamento (Jenaabadi et al., 2017; Ko, 2017). Um estudo conduzido em Singapura, observou que estudantes com maior desenvolvimento de relações interpessoais e/ou religiosas mostraram-se mais aptos a resistir a depleção emocional, visto que possuíam mecanismos de escape das atividades acadêmicas, representados em seus amigos, colegas e familiares (Chue \& Cheung, 2021).

No que se refere ao conhecimento sobre saúde sexual e reprodutiva, esse é adquirido pelo estudo da Anatomia e Fisiologia dos sistemas genitais feminino e masculino, durante o período escolar do ensino básico. Em virtude da inexistência de programas de educação sexual para jovens, o conhecimento relativo sobre a própria sexualidade ainda é limitado (Zeglin et al., 2018). Embora possa haver uma relação positiva entre educação sexual e a probabilidade de ocorrência de relações libidinosas, o risco que envolve o ato sexual seria bastante reduzido. Isso porque, com o autoconhecimento e autonomia os jovens podem se proteger de doenças e da gravidez indesejada (Li et al., 2017).

Nesse contexto, é notório que o conhecimento sobre a função sexual está associado não só ao aspecto físico, mas, de forma mais importante, ao emocional e psicológico dos indivíduos. Assim, a sexualidade é fator importante para qualidade de vida, uma vez que trata o indivíduo como pessoa que apresenta fragilidades emocional, física e psicológica. Portanto, um indivíduo que exerce sua sexualidade de forma holística é mais alegre (Zamboni \& Zaid, 2017).

Ainda assim, é importante ressaltar as diferenças entre os sexos, pois homens por uma herança histórica/cultural e biológica, apresentam prazer físico, ou seja, possuem uma sexualidade sinestésica (tato, olfato, visão e audição), já as mulheres, atingem o prazer por meio das emoções (Silva et al., 2021; Souza Junior et al., 2021). E, pensando nisso, Chung et al. (2021) constataram que mulheres apresentam maiores taxas de disfunção sexual, decorrente da incapacidade do parceiro durante as relações e/ou ao próprio desconhecimento do corpo.

Percebe-se uma existência de pré-conceitos quanto à personalidade de indivíduos com vida sexual ativa e, porém, ausente, principalmente durante o período da juventude e idade adulta. O consumo de bebidas alcoólicas e tabaco, além de participação em festas, atividades comuns a qualquer acadêmico durante o momento de faculdade, relacionam-se a uma vida 
sexual ativa e de risco. No entanto, frequentar centros religiosos e se abster de atividades festivas associam-se a vida sexual ausente (Singh, 2020). Serinolli e Novaretti (2017) avaliaram a QV dos acadêmicos na escola de medicina de São Paulo e constataram que a espiritualidade e crenças religiosas impactaram de forma importante a saúde emocional. Identificaram menor incidência de afecções psicológicas como depressão e ansiedade nos acadêmicos que relataram algum tipo de crença.

Ao se avaliar aspectos sociodemográficos e sua relação com os aspectos emocionais, é perceptível que o sexo feminino demonstra maior inteligência emocional, por isso conseguem perceber, compreender e regular melhor suas emoções. O sexo masculino, no entanto, mostra-se incapaz de manter uma boa regulação emocional em situações de estresse (Garner \& Waajid, 2019). Em contrapartida, Hajian-Tilaki et al. (2017) relataram que mulheres apresentaram pior qualidade de vida, não por um fator biológico, mas em razão de aspectos multifatoriais, como a renda salarial inferior e menor nível de escolaridade em geral. Nesse contexto, Ramos-Lima et al. (2018) descreveram a escolaridade como fator que pode influenciar a saúde emocional dos indivíduos, sendo o baixo nível educacional relacionado a baixa adesão e manutenção de um estilo de vida saudável, física e psicologicamente.

Para que haja maior esclarecimento entre os diversos fatores que influenciam a saúde emocional e suas potenciais consequências, são necessários mais estudos acerca dessa temática, sobretudo com amostras populacionais diferentes e maiores, com o controle de possíveis vieses para que possa corroborar com mais certeza o impacto desses fatores na vida acadêmica e pessoal do estudante. Contudo, os dados já existentes podem ser usados como base para orientar futuras pesquisas científicas e tomada de decisões na busca por soluções ao estresse da vida acadêmica, em especial o estudante de medicina ou da área da saúde, que por vezes prejudica a própria saúde em busca do conhecimento para cuidar da saúde do próximo.

\section{Considerações Finais}

Os resultados apresentados neste estudo, mostram os principais fatores emocionais, e outros aspectos que podem influenciar na qualidade de vida dos acadêmicos da área da saúde, em especial os de medicina. Foram apresentados, ainda, diversos componentes que colaboram para o surgimento de uma instabilidade emocional, dentre as quais destaca-se a própria pressão do cotidiano universitário, que por sua vez culmina com o baixo rendimento na aprendizagem.

No entanto, apesar de todos os efeitos negativos do esgotamento emocional, esses componentes podem ser gerenciados com a ajuda da Medicina Preventiva no nível da atenção primária, como nas unidades de apoio ao estudante, já presente em muitas universidades, com a promoção e manutenção da saúde mental, por meio de educação psicológica aos universitários. É importante que esses aconselhamentos sejam acompanhados da estimulação na continuidade dos estudos e da desestimulação de mecanismos prejudiciais à saúde como a falta de descanso para recuperar horas de estudos. Além disso, seria essencial o engajamento conjunto do governo, que poderia incentivar mais esforços para a educação física e hábitos de vida saudáveis entre os estudantes universitários, o que colaboraria na diminuição do esgotamento emocional nessa população.

Além disso, os resultados mostraram que são evidentes os fatores que indicam sofrimento mental, como a falta de motivação nos estudos, a dificuldade em manter atenção, a incapacidade de completar tarefas e, até mesmo, a falta de interesse em se relacionar com novas pessoas. Com isso, é importante utilizar esses fatores como sinais de alerta e sempre procurar manter boas relações interpessoais como meio de apoio e estímulo ao desenvolvimento de habilidades sociais. Quanto à função sexual, é preciso ampliar as propostas de educação sobre o tema na educação básica, com a finalidade de diminuir, tanto os riscos que envolvem o ato sexual como riscos de transmissão de doenças, quanto os que envolvem à saúde da função sexual humana e o conhecimento relativo a própria sexualidade. 


\section{Referências}

Andrade, F. K., Caetano, L. A. O., Oliveira, W. A., Silva, J. L., \& Manochio-Pina M. G. (2019). Qualidade de vida e burnout entre estudantes de medicina que vivenciam o método de Aprendizagem Baseada em Problemas. Aletheia, 52(1), 128. 116- DOI: DOI 10.29327/226091

Anversa, A. C., Santos Filha, V. A. V., Silva, E. B., \& Fedosse, E. (2018). Qualidade de vida e o cotidiano acadêmico: uma reflexão necessária. Cadernos Brasileiros de Terapia Ocupacional, 26(03), 626-631. DOI: https://doi.org/10.4322/2526-8910.ctoAO1185

Araújo, D. C., Almeida, C. P., Santana, L. R. P., Mota, S. B., Santos, A. D., Lima, S. V. M. A., Araújo, K. C. G. M., Alves, J. A. B., \& Vaez, A. C. (2021). Qualidade de vida dos estudantes da área da saúde que utilizam metodologia ativa de ensino-aprendizagem. Research, Society and Development, 10(5), e15410514737. DOI: https://doi.org/10.33448/rsd-v10i5.14737

Bezerra, M. P. P., Lira, R. C. M., Silva, A. O., Melo, I. L. P., Silva, N. S., \& Roque, T. S. (2020). A influência da variação no ciclo do sono sobre o desempenho universitário e o bem-estar de estudantes de Medicina. Research, Society and Development, 9(12), e28091211057. DOI: https://doi.org/10.33448/rsdv9i12.11057

Brodski, S. K., \& Hutz, C. S. (2012). The Repercussions of Emotional Abuse and Parenting Styles on Self-Esteem, Subjective Well-Being: A Retrospective Study with University Students in Brazil, Journal of Aggression, Maltreatment \& Trauma, 21:3, 256-276, DOI: 10.1080/10926771.2012.666335

Cazolari, P. G., Cavalcante, M. S., Demarzo, M. M. P., Cohrs, F. M., Sanudo, \& A., Schveitzer, M. C. (2020) Burnout and Well-Being Levels of Medical Students: a Cross-Sectional Study. Revista Brasileira de Educação Médica, 44(04), e125. DOI: https://doi.org/10.1590/1981-5271v44.4-20190138

Chazan, A. C. S., Campos, M. R., \& Portugal, F. B. (2015). Qualidade de vida de estudantes de medicina da UERJ por meio do Whoqol-bref: uma abordagem multivariada. Ciência \& Saúde Coletiva, 20(2), 547-556. DOI: https://doi.org/10.1590/1413-81232015202.05182014

Christ, C., de Waal, M. M., Dekker, J., van Kuijk, I., van Schaik, D., Kikkert, M. J., Goudriaan, A. E., Beekman, A., \& Messman-Moore, T. L. (2019). Linking childhood emotional abuse and depressive symptoms: The role of emotion dysregulation and interpersonal problems. PloS one, 14(2), e0211882. DOI: https://doi.org/10.1371/journal.pone.0211882

Chue, J. S. X., \& Cheung, H. S. (2021). Mental resilience enhances the well-being of Singaporean college students through reducing burnout. Current Psychology, $1-10$.

Chung, M. H., Chao, J. K., Ma, M. C., \& Lin R. W. (2021). Sexuality and Quality of Life in Eastern Taiwan People With Schizophrenia, 23 July 2021, PREPRINT (Version 1) available at Research Square. DOI: https://doi.org/10.21203/rs.3.rs-514841/v1

Cruz, M. C. A., Garcia, T. R., Macedo, R. M., Freitas, Y. J. F., Borges, N. M. P., Silva, A. C. S. P., Silva, M. L., \& Arruda, J. T. (2021). Influência na qualidade de vida dos estudantes de Medicina relacionadas a má alimentação e sono. Research, Society and Development, 10(2), e23710212393. DOI: https://doi.org/10.33448/rsd-v10i2.12393

Dutra, L. L., Aquino, A. C. N., Lima da Silva, E., \& Barros, L. N. (2021). Avaliação do Índice de Qualidade do Sono de Pittsburgh em estudantes de Medicina: Uma revisão integrativa da literatura. Research, Society and Development, 10(8), e52410817530. https://doi.org/10.33448/rsd-v10i8.17530

El-Ibiary, S. Y., Yam, L., \& Lee, K. C. (2017). Assessment of Burnout and Associated Risk Factors Among Pharmacy Practice Faculty in the United States. American journal of pharmaceutical education, 81(4), 75. https://doi.org/10.5688/ajpe81475

Ferreira, M. A. A., Domingos, S. R. A., Trovão, C. B. A., Domiciano, D. C., Silva, B. A., Rocha, L. G., Carmello, L. M., \& Soares, E. A. (2020). Perfil da Qualidade de Sono de um grupo de estudantes de Medicina. Research, Society and Development, 9(9), e718997806. DOI: https://doi.org/10.33448/rsd-v9i9.7806

Garner, P. W., \& Waajid, B. (2019). Sociodemographic variations in the linkage between emotion regulation and peer victimization, Journal of School Violence, 18:1, 121-133, DOI: 10.1080/15388220.2017.1418670

Grace, M. K. (2018). Depressive symptoms, burnout, and declining medical career interest among undergraduate pre-medical students. International journal of medical education, 9, 302-308. DOI: https://doi.org/10.5116/ijme.5be5.8131

Hajian-Tilaki, K., Heidari, B., \& Hajian-Tilaki, A. (2017). Are Gender Differences in Health-related Quality of Life Attributable to Sociodemographic Characteristics and Chronic Disease Conditions in Elderly People? International journal of preventive medicine, 8, 95. DOI: https://doi.org/10.4103/ijpvm.IJPVM_197_16

Im, H.-J., \& Lee, Y. L. (2021). A study of the relationship between learning flow and learning burnout in college online classes. Journal of Digital Convergence, 19(6), 39-46. DOI: https://doi.org/10.14400/JDC.2021.19.6.039

Jenaabadi, H., Nastiezaie, N., \& Safarzaie, H. (2017). The relationship of academic burnout and academic stress with academic self-efficacy among graduate students. The New Educational Review, 49(3):65-76. DOI: 10.15804/tner.2017.49.3.05

Ko, C. M. (2017). Effects of empowerment and emotional intelligence in the relationship between clinical practicum stress and burnout among nursing college students. Korean Journal of Stress Research, 25(2), 120-127. DOI:10.17547/KJSR.2017.25.2.120

Leite, J. T. S., Leite, O. D., Rodrigues, J. W. S., Mendes, V. L., Moreira, A. F. C. M., Costa Neto, A. F., Xerez, M. C., Pinheiro, J. C., Paiva, D. F. F., \& Carolino, R. de A. (2020). Avaliação dos sintomas de estresse e qualidade de vida dos estudantes de medicina do alto sertão paraibano. Research, Society and Development, 9(11), e89891110487. DOI: https://doi.org/10.33448/rsd-v9i11.10487

Li, C., Cheng, Z., Wu, T., Liang, X., Gaoshan, J., Li, L., Hong, P., \& Tang, K. (2017). The relationships of school-based sexuality education, sexual knowledge and sexual behaviors-a study of 18,000 Chinese college students. Reproductive health, 14(1), 103. DOI: https://doi.org/10.1186/s12978-017-0368-4

Madu, S. N. (2003). The relationship between parental physical availability and child sexual, physical and emotional abuse: a study among a sample of university students in South Africa. Scandinavian journal of psychology, 44(4), 311-318. DOI: https://doi.org/10.1111/1467-9450.00350 
Mazucato, J. B., Ferrarezi, F. C., Lima, L. C., Favaro, P. F., \& Pozo, M. (2020). Investigação sobre a influência da cefaleia na qualidade de vida dos estudantes de diferentes anos do curso de medicina de uma instituição de São José do Rio Preto. Research, Society and Development, 9(12), e44291211441. DOI: https://doi.org/10.33448/rsd-v9i12.11441

Mceachern, A. G., Aluede, O., \& Kenny, M. C. (2008). Emotional abuse in the classroom: Implications and interventions for counselors. Journal of Counseling \& Development, 86(1), 3-10. DOI: 10.1002/j.1556-6678.2008.tb00619.x

Meinck, F., Cosma, A. P., Mikton, C., \& Baban, A. (2017). Psychometric properties of the Adverse Childhood Experiences Abuse Short Form (ACE-ASF) among Romanian high school students. Child abuse \& neglect, 72, 326-337. DOI: https://doi.org/10.1016/j.chiabu.2017.08.016

Mendes, T. C., \& Dias, A. C. P. (2021). Sintomas de depressão, ansiedade, estresse e fatores associados em estudantes de medicina brasileiros: revisão integrativa. Research, Society and Development, 10(4), e14910414033. DOI: https://doi.org/10.33448/rsd-v10i4.14033

Meyer, C., Barbosa, D. G., Andrade, R. D., Ferrari Junior, G. J., Gomes Filho Neto, M., Guimarães, A. C., \& Felden, E. P. G. (2019). Qualidade de vida de estudantes de medicina e a dificuldade de conciliação do internato com os estudos. ABCS Health Sciences, 44(2), $108-113$.

Mitra, S., Sarkar, A. P., Haldar, D., Saren, A. B., Lo, S., \& Sarkar, G. N. (2018). Correlation among perceived stress, emotional intelligence, and burnout of resident doctors in a medical college of West Bengal: A mediation analysis. Indian journal of public health, 62(1), 27-31. DOI: https://doi.org/10.4103/ijph.IJPH_368_16

Ramos-Lima, M., Brasileiro, I. C., Lima, T. L., \& Braga-Neto, P. (2018). Quality of life after stroke: impact of clinical and sociodemographic factors. Clinics (Sao Paulo, Brazil), 73, e418. DOI: https://doi.org/10.6061/clinics/2017/e418

Rich, D. J., Gingerich, K. J., \& Rosen, L. A. (1997). Childhood Emotional Abuse and Associated Psychopathology in College Students, Journal of College Student Psychotherapy, 11:3, 13-28, DOI: 10.1300/J035v11n03_04

Santos, L. S. Ribeiro, I. J. S., Boery, E. N., \& Boery R. N. S. O. (2017). Qualidade de vida e transtornos mentais comuns em estudantes de medicina. Cogitare Enfermagem, 22(4), e52126. DOI: http://dx.doi.org/10.5380/ce.v22i4.52126

Serinolli, M. I., \& Novaretti, M. (2017). A cross-sectional study of sociodemographic factors and their influence on quality of life in medical students at Sao Paulo, Brazil. PloS one, 12(7), e0180009. DOI: https://doi.org/10.1371/journal.pone.0180009

Shrestha, D. B., Katuwal, N., Tamang, A., Paudel, A., Gautam, A., Sharma, M., Bhusal, U., \& Budhathoki, P. (2021). Burnout among medical students of a medical college in Kathmandu; A cross-sectional study. PLOS ONE 16(6): e0253808. DOI: https://doi.org/10.1371/journal.pone.0253808

Silva, A. C. S. P., Mori, A. S., Silva, M. L., Cruz, M. C. A., Borges, N. M. P., Freitas, Y. J. F., Garcia, T. R., Macedo, R. M., \& Arruda, J. T. (2021). Saúde sexual feminina em tempos de empoderamento da mulher. Research, Society and Development, 10(7), e28010716415. DOI: http://dx.doi.org/10.33448/rsdv10i7.16415

Silva, M. L., Silva, M. L., Silva, A. C. S. P., Freitas, Y. J. F., Borges, N. M. P., Cruz, M. C. A., Mori, A. S., Macedo, R. M., Garcia, T. R., \& Arruda, J. T. (2020). Condições que interferem na qualidade de vida do estudante de Medicina. Research, Society and Development, 9(11), e2469119640. DOI: https://doi.org/10.33448/rsd-v9i11.9640

Singh, J. K. (2020). An Empirical Study of Alcoholic versus Nonalcoholic Person. International Journal of Recent Advances in Psychology \& Psychotherapy, $4(1), 20-25$.

Souza Junior, E. V., Silva Filho, B. F., Barros, V. S., Souza, A. R., Cordeiro, J. R. J., Siqueira, L. R., Sawada, N. O. (2021). Sexuality is associated with the quality of life of the elderly! Revista Brasileira de Enfermagem, v. 74(suppl 2), e20201272. DOI: https://doi.org/10.1590/0034-7167-2020-1272

Spadine, M., Patterson, M. S., Brown, S., Nelon, J., Lanning B., \& Johnson, D. M. (2020). Predicting emotional abuse among a sample of college students, Journal of American College Health, 1-9. DOI: 10.1080/07448481.2020.1740709

Vidourek, R. A. (2017). Emotional abuse: Correlates to abuse among college students. Journal of Aggression, Maltreatment \& Trauma, 26:7, 792-803, DOI: $10.1080 / 10926771.2017 .1308980$

Wang, Q., Shi, W., \& Jin, G. (2019). Effect of Childhood Emotional Abuse on Aggressive Behavior: A Moderated Mediation Model, Journal of Aggression, Maltreatment \& Trauma, 28:8, 929-942, DOI: 10.1080/10926771.2018.1498962

WHO, World Health Organization. (2012). Programmeon mental health: WHOQOL user manual, 2012 revision.

Zamboni, B. D., \& Zaid, S. J. (2017). Human sexuality education in marriage and family therapy graduate programs. Journal of Marital and Family Therapy, 43(4), 605-616. DOI: https://doi.org/10.1111/jmft.12214

Zeglin, R. J., Van Dam, D. \& Hergenrather, K. C. (2018). An Introduction to Proposed Human Sexuality Counseling Competencies. International Journal for the Advancement of Counselling 40, 105-121. DOI: https://doi.org/10.1007/s10447-017-9314-y 